\title{
Expert Discussion: Ductal Carcinoma in situ
}

\author{
Peter Dall $^{\mathrm{a}}$ Jörg Heil ${ }^{\mathrm{b}}$ Maggie Banys-Paluchowskic ${ }^{\mathrm{C}}$ David Krug $^{\mathrm{d}}$ \\ Wilfried Budach ${ }^{\mathrm{e}}$ Cornelia Kolberg-Liedtke ${ }^{f}$ Toralf Reimer $^{g}$

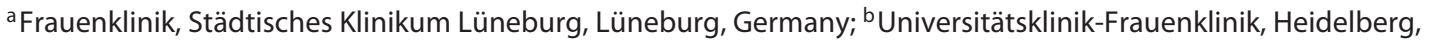 \\ Germany; ${ }^{C}$ Klinik für Gynäkologie und Geburtshilfe, Universitätsklinikum Schleswig-Holstein Campus Lübeck, \\ Lübeck, Germany; ${ }^{\mathrm{d} D e p a r t m e n t}$ of Radiation Oncology, University Hospital Schleswig Holstein, Kiel, Germany; \\ ${ }^{e}$ Department of Radiation Oncology, Heinrich Heine University, Düsseldorf, Germany; ${ }^{f}$ Klinik für Frauenheilkunde \\ und Geburtshilfe, Universitätsklinikum Essen, Essen, Germany; ${ }^{9}$ Universitätsfrauenklinik am Klinikum Südstadt, \\ Klinikum Südstadt Rostock, Rostock, Germany
}

\section{Dall: Multicentric Microcalcification - Optimal Noninvasive and Minimally Invasive Diagnostic Procedure}

Heil: In case of multicentric microcalcification only, we should aim for a stereotactically guided vacuumassisted biopsy of the most suspicious region first. In case of a pathological diagnosis of invasive cancer, ductal carcinoma in situ (DCIS), atypical ductal hyperplasia or other B3 lesions, it is necessary to biopsy another region of microcalcification which should be in another quadrant of the breast in order to clearly define the extent of surgery. Multicentric, i.e. over more than a quadrant, disease should be treated by mastectomy which can be skin or even nipple sparing if the patient wishes a breast reconstruction.

\section{Dall: Operative Treatment - Is There Still a Maximum} DCIS Extent to Accept Breast-Conserving Operation?

Banys-Paluchowski: While operative options have improved considerably in the past decades due to implementation of oncoplastic breast-conserving surgery (BCS), the therapy of extensive DCIS remains challenging. The following aspects need to be taken into account when discussing surgical options: the suspected extent of disease upon imaging, breast volume and form (e.g., ptosis), tumor-nipple distance and the patient's wishes. In case of large breasts, resection of extensive DCIS may be combined with oncoplastic reduction mammoplasty. However, extensive DCIS in patients with smaller breasts often requires a mastectomy with or without immediate reconstruction.

Heil: No, a metric DCIS extent cannot be defined to accept breast-conserving surgery. A $10-\mathrm{cm}$ linear, segmental extension in the upper outer quadrant in a large breast could be managed with breast conservation in one patient while a $3-\mathrm{cm}$ diffuse extension of the inner lower quadrant in a small breast might not be a good candidate for breast-conserving surgery.

\section{Dall: Pre- and Perioperative Options to Reduce the Incidence of Incomplete (R1) DCIS Resections}

Banys-Paluchowski: Careful planning is essential to ensure complete resection at surgery. This requires an interdisciplinary approach involving both the surgeon and the diagnostician. Preoperative localization is necessary in most cases and includes a placement of a wire or a special marker. These include but are not limited to magnetic or radioactive seeds, radar markers and radiofrequency identification tags that can be detected using a special probe during surgery. In case of extensive lesions, use of multiple markers or bracketing wires is recommended. A specimen radiogram should be performed to ensure complete resection of the lesion. In case of close 
margins an immediate re-excision is recommended. Since frozen sections are not reliable in case of DCIS, they are usually not performed.

Heil: Possibly incomplete in situ resections, either with or without invasive cancer, should be handled individually. Pre- and perioperative options to reduce unclear or positive margins are: (1) individual assessment and selection of adequate imaging and minimally invasive biopsies to define as well as possible the extent of the DCIS; (2) preoperative location by wires, seed or other location techniques to determine the extent of resection, in case with an anticipated extension of more than $3 \mathrm{~cm}$ with bracketing techniques; (3) ability of the breast surgeon to read the imaging before and during the operation properly and plan the surgery accordingly; (4) intraoperative specimen radiography; (5) techniques like MarginProbe can be used intraoperatively.

\section{Dall: Irradiation after Breast-Conserving DCIS Operation - Nil, Hypofractionated, Conventionally Fractionated, Partial Breast Irradiation?}

Krug/Budach: Radiotherapy for patients with DCIS is no longer a one-size-fits-all approach. While observation after BCS for DCIS with low-risk features (tumor size $<2.5 \mathrm{~cm}, \mathrm{G} 1-2$, margin $\geq 2 \mathrm{~mm}$ ) is considered appropriate by most authors, updated results from the RTOG 9804 trial have recently shown that even in this subgroup, whole-breast radiotherapy reduced local recurrences and invasive recurrences, which occurred in 15 and $10 \%$ of patients at 15 years in the observation arm, by more than $50 \%$ [1]. While moderate hypofractionation has already replaced conventional fractionation as the standard of care in patients with invasive breast cancer, this question remained unanswered for patients with DCIS in the absence of randomized controlled trials. However, with the presentation of the BIG 3-07/TROG 07.01 trial at SABCS 2020, moderate hypofractionation can be considered an equally safe and effective option for patients with DCIS [2]. Due to the decreased acute toxicity and patient burden, this will surely lead to change in clinical practice. Another option to individualize adjuvant radiotherapy for patients with low-risk DCIS is partial breast irradiation which treats only the lumpectomy cavity with an additional safety margin. Both the RAPID and the NSABP B-39 trials which tested external-beam partial breast irradiation included sufficient numbers of patients with DCIS ( $>350$ and $>1,000$ patients, respectively) and demonstrated similar rates of local recurrence as compared to wholebreast radiotherapy. Thus, this has been included as a treatment option in the DEGRO and AGO guidelines for patients with low-risk DCIS $[3,4]$.
Last but not least, there is the option to deliver an additional tumor bed boost in patients at increased risk of local recurrence. The above-mentioned BIG 3-07/TROG 07.01 trial [2] included a second randomization to an additional tumor bed boost of $16 \mathrm{~Gy}$ delivered in 8 fractions. This decreased the local recurrence rate at 5 years by $4 \%$, but also increased acute and late toxicity, although highgrade toxicity was rare. Thus, a tumor bed boost may be considered in patients with high-risk features similar to invasive breast cancer utilizing a shared decision-making approach.

\section{Dall: Is There Still an Indication for Systemic Endocrine Treatment after Operation and Irradiation - When and Why?}

Kolberg-Liedtke: It is important to acknowledge that on the one hand DCIS represents a disease that at the time of diagnosis is not life-threatening and that on the other hand in case of recurrence, less than one third will become invasive and carry the potential to form lifethreatening metastases. In fact, adjuvant endocrine therapy of DCIS is aimed at reducing particularly contralateral and to a lesser extent ipsilateral (non)invasive recurrences and has no value in improving overall survival. Numbers needed to treat for prevention of invasive and noninvasive recurrence by administration of tamoxifen are around 15 and 59, respectively, and as many as 29 patients needed to be treated with aromatase inhibitors to prevent 1 invasive disease recurrence. On the other hand, use of both tamoxifen and aromatase inhibitors may be associated with significant toxicities such as thrombosis, endometrial cancer and osteoporosis, respectively.

Consequently, the AGO does not recommend routine adjuvant therapy with either tamoxifen or aromatase inhibitor (+/-) and stresses that endocrine therapy should only be indicated in certain risk constellations in accordance with patient preference [3]. However, if definitive risk factors that identify a high enough risk of recurrence and justify routine endocrine therapy in a defined group of patients with DCIS are lacking, the decision should be made by the patient as part of an informed-consent process. The treating physician should balance the risks (side effects) against the potential benefits (reduction in the risk of recurrence) and allow the patient to come to an individualized decision as much as possible. To my experience, only a small number of patients will opt to take endocrine therapy for increased local control alone; nevertheless, each suitable patient should decide as independently as possible whether she feels her fear of experiencing disease recurrence to be high enough to justify the risks of significant side effects. 
Reimer: Over the last few years the prescription of endocrine therapy after BCS and irradiation for DCIS significantly decreased in Germany. Currently, the rate for adjuvant endocrine treatment in this setting is less than $10 \%$ at our breast unit in Rostock. The reasons for controversial discussion regarding risk and benefit of endocrine systemic therapy are:

- an overall survival advantage has not been demonstrated;

- the number needed to treat (for every in-breast recurrence) is 15 according to the AGO breast guideline statement;

- there are several side effects for used aromatase inhibitors (only in the postmenopausal situation) and tamoxifen: increased numbers for osteoporosis, fractures and cerebrovascular accidents with aromatase inhibitors, risk for the development of gynecological cancers due to tamoxifen;

- the effect on event-free survival is more visible in patients with BCS and without postoperative radiotherapy;

- patients with DCIS has a favorable prognosis (recurrence rate less than $10 \%$ after 15 years of follow-up in the IBIS-II DCIS trial), and the great majority of this entity is likely a nonobligate precursor for invasive cancer.

However, available published data suggest endocrine therapy provides risk reduction in the ipsilateral breast after BCS and in the contralateral breast in patients treated with mastectomy or BCS with estrogen receptor (ER)positive DCIS. Especially the ipsilateral benefit was seen only in patients with ER-positive DCIS according to NSABP B-24 results. Based on this, the ASCO Expert Panel recommends ER testing in DCIS to guide the discussion about adjuvant endocrine therapy. Data on whether progesterone receptor testing in DCIS adds predictive or prognostic value beyond that of ER alone are currently lacking.

In conclusion, benefits must be balanced against side effects of endocrine treatment as individual consideration. The decision to use endocrine therapy will depend on patient goals and discussion with their clinical care team, but patients should be informed about primary risk reduction options in ER-positive DCIS.

\section{Conflict of Interest Statement}

Peter Dall: honoraria for lectures and advisory boards: Roche, Novartis, Pfizer, Lilly, AstraZeneca, Daiichi Sankyo, Seagen. Jörg Heil: no conflict of interest to declare. Maggie Banys-Paluchowski: honoraria for lectures and advisory boards: Roche, Novartis, Pfizer, Lilly, Eisai, AstraZeneca, Amgen, GSK, pfm, Daiichi Sankyo. Study support: EndoMag, Mammotome, MeritMedical. David Krug: received honoraria from Merck Sharp \& Dohme, the Euro- pean School of Oncology and the European Society for Medical Oncology. Wilfried Budach: no conflict of interest to declare. Cornelia Kolberg-Liedtke: no conflict of interest to declare. Toralf Reimer: no conflict of interest to declare.

\section{Chair}

Prof. Peter Dall

Chefarzt Frauenklinik

Städtisches Klinikum Lüneburg gemeinnützige $\mathrm{GmbH}$

Bögelstraße 1

DE-21339 Lüneburg (Germany)

frauenklinik@klinikum-lueneburg.de

\section{Participants}

Prof. Jörg Heil

Zentrum für Frauenheilkunde

Im Neuenheimer Feld 440

DE-69120 Heidelberg (Germany)

joerg.heil@med.uni-heidelberg.de

PD Dr. Maggie Banys-Paluchowski

Klinik für Gynäkologie und Geburtshilfe

Universitätsklinikum Schleswig-Holstein Campus Lübeck

Ratzeburger Allee 160

DE-23562 Lübeck (Germany)

maggiebanys@yahoo.de

\section{Dr. David Krug}

Klinik für Strahlentherapie

Campus Kiel

Arnold-Heller-Strasse 3, Haus L

DE-24105 Kiel (Germany)

david.krug@uksh.de

\section{Prof. Wilfried Budach}

Klinik für Strahlentherapie und Radioonkologie

Moorenstrasse 5

DE-40225 Düsseldorf (Germany)

Wilfried.Budach@med.uni-duesseldorf.de

Prof. Cornelia Kolberg-Liedtke

Klinik für Frauenheilkunde und Geburtshilfe

Universitätsklinikum Essen

Hufelandstraße 55

DE-45147 Essen (Germany)

cornelia1979@googlemail.com

\section{Prof. Toralf Reimer}

Universitäts-Frauenklinik Rostock am Klinikum Südstadt Südring 81

DE-18059 Rostock (Germany)

toralf.reimer@med.uni-rostock.de 


\section{References}

1 McCormick B, Winter KA, Woodward W, Kuerer HM, Sneige N, Rakovitch E, et al. Randomized phase III trial evaluating radiation following surgical excision for good-risk ductal carcinoma in situ: long-term report from NRG Oncology/RTOG 9804. J Clin Oncol. 2021;JCO.21.01083. Epub 2021 Aug 18.
2 Chua BH, Link E, Kunkler I, Olivotto I, Westenberg $\mathrm{AH}$, Whelan $\mathrm{T}$, et al. A randomized phase III study of radiation doses and fractionation schedules in non-low risk ductal carcinoma in situ (DCIS) of the breast (abstract GS2-04). BIG 3-07/TROG 07.01 Gen Sess Abstr. 2021;GS2-04-GS2-04.

3 Ditsch N, Kolberg-Liedtke C, Friedrich M, Jackisch C, Albert US, Banys-Paluchowski M, et al. AGO recommendations for the diagnosis and Tteatment of patients with early breast cancer: update 2021. Breast Care (Basel). 2021 Jun;16(3):214-27.
4 Strnad V, Krug D, Sedlmayer F, Piroth MD, Budach W, Baumann R, et al; Breast Cancer Expert Panel of the German Society of Radiation Oncology (DEGRO). DEGRO practical guideline for partial-breast irradiation. Strahlenther Onkol. 2020 Sep;196(9):749-63. 